\title{
Resection of large recurrent third ventricular epidermoid tumors through the posterior interhemispheric transcallosal approach
}

\author{
Benjamin K. Hendricks, BS, and Aaron A. Cohen-Gadol, MD, MSc
}

Goodman Campbell Brain and Spine and Indiana University Department of Neurological Surgery, Indianapolis, Indiana

Epidermoid cysts or tumors comprise $1 \%$ of intracranial tumors with a predilection for the cerebellopontine angle or parasellar cisterns. These tumors are derived from an ectopic ectodermal implant seeded between fusing ectodermal surfaces. This results in a benign proliferation of stratified squamous epithelium along a cyst wall with a lumen composed of hyperkeratotic material derived from the desquamating cells. Surgical intervention is the treatment of choice for symptomatic patients. There is no role for radiotherapy or chemotherapy in the treatment of these lesions, including for residual or recurrent disease. The inherent adhesion of the proliferating epithelial lining to surrounding neurovascular structures significantly complicates attempts at gross-total resection. Epidermoid tumors or cysts are rarely reported within the third ventricular region. In this video, the authors demonstrate the technique of right-sided posterior interhemispheric transcallosal approach for maximal resection of a complex recurrent third ventricular epidermoid tumor with lateral ventricular extension.

The video can be found here: https://youtu.be/wbXp7mc0vT4.

KEYWORDS epidermoid; interhemispheric transcallosal; third ventricle; video 